\title{
Malte de variedades de amaranto
}

\author{
Patrícia Monique CRIVELARI-COSTA ${ }^{*}$ \& Aloisio BIANCHINI ${ }^{1}$
}

\author{
${ }^{1}$ Universidade do Estado de Mato Grosso, Sinop, MT, Brasil. \\ ${ }^{2}$ Programa de Pós-Graduação em Agricultura Tropical, Universidade Federal de Mato Grosso, Cuiabá, MT, Brasil. \\ *E-mail: patriciacrivellari@gmail.com \\ (Orcid: 0000-0002-6362-617X; 0000-0002-1225-45X)
}

\begin{abstract}
Recebido em 12/12/2021; Aceito em 17/12/2021; Publicado em 18/12/2021.
RESUMO: Objetivou-se obter e avaliar o malte de amaranto, das espécies Amaranthus cruentus, com as variedades BRS Alegria e Verde, e Amaranthus caudatus, com a variedade Inca, produzidas no cerrado matogrossense. Foi realizado teste de germinação com alternância no fotoperíodo. A qualidade do malte produzido foi avaliada pela análise do poder diastático e o rendimento. Observou-se, pela análise de fotoblastismo, que a variedade Verde é fotoblástica neutra e BRS Alegria e Inca são pouco tolerantes à luz constante. O poder diastático, medido pelo índice WK variou em função da temperatura e variedades sendo que a variedade Inca teve maior rendimento e poder diastático $(187,4 \mathrm{WK})$ à temperatura de $25^{\circ} \mathrm{C}$; a temperatura de $25^{\circ} \mathrm{C}$ produziu maltes com maior poder diastático, da ordem de 168,4 WK; A variedade BRS Alegria teve menor poder diastático, sendo menos indicada para a produção de malte para fins cervejeiros. Os resultados foram satisfatórios para a produção de bebidas lácteas maltadas, no entanto, para fins cervejeiros, novos ensaios de maltagem devam ser conduzidos, na tentativa de elevar o poder diastático do malte deste pseudocereal para valores próximos do mínimo recomendado para este fim, que é de $220 \mathrm{WK}$.
\end{abstract}

Palavras-chave: Amaranthus cruentus; Amaranthus caudatus; BRS Alegria; fotoblastismo; poder diastático.

\section{Amaranth varieties malt}

\begin{abstract}
The objective of this study was to obtain and evaluate malt amaranth, of the species $A$ maranthus cruentus, with BRS Alegria and Verde varieties, and Amaranthus caudatus, with Inca variety. Germination test was performed with alternating photoperiod. The quality of the malt produced was evaluated by analyzing the diastatic power and the yield. It was observed, by the analysis of photoblastism, that the Verde variety is photoblastic neutral and BRS Alegria and Inca are poorly tolerant to constant light. Diastatic power, measured by the WK index, varied as a function of temperature and varieties, with the Inca variety having higher yields and diastatic power $(187.4 \mathrm{WK})$ at a temperature of $25{ }^{\circ} \mathrm{C}$; the temperature of $25{ }^{\circ} \mathrm{C}$ produced malts with greater diastatic power, in the order of $168.4 \mathrm{WK}$; The BRS-Alegria variety had lesser diastatic power, being less suitable for the production of malt for brewing purposes. The results were satisfactory for the production of malted dairy beverages, however, for brewing purposes, new malting tests should be conducted, in an attempt to raise the diastatic power of the malt of this pseudocereal to values close to the minimum recommended for this purpose, which is $220 \mathrm{WK}$.
\end{abstract}

Keywords: Amaranthus cruentus; Amaranthus caudatus; BRS Alegria; photoblastism; diastatic power.

\section{INTRODUÇÃO}

O amaranto é um pseudocereal da classe das dicotiledôneas, pertencente ao gênero Amaranthus, família Amaranthaceae e é conhecido como uma promissora matériaprima pelo seu excepcional valor nutricional, além de não conter glúten em sua estrutura, o que permite $\mathrm{o}$ desenvolvimento de formulações de alimentos para pacientes com doença celíaca (PAGAMUNICI et al., 2014). As plantas pertencentes a essas espécies, adapta-se rapidamente a condições ambientais adversas, como déficit hídrico e altas temperaturas e são fáceis de cultivar (SILVA et al., 2019; ZHANG et al., 2019).

A incorporação do sementes de amaranto como ingrediente em preparações culinárias e produtos alimentícios tem sido explorada por apresentar o atrativo de possuir alta densidade calórica e baixa viscosidade, esta última a partir do processamento com formação de malte de amaranto, obtido através da germinação da semente (FERREIRA et al., 2007; MOTTA et al., 2019).
Os processos germinativos são resultados das características fisiológicas da própria semente e das condições ambientais do agroecossistema, promovendo uma sequência de atividades metabólicas, que resulta na retomada do desenvolvimento do eixo embrionário (BEWLEY et al., 2012). Sementes de diferentes espécies apresentam respostas diferenciadas à germinação quanto à temperatura e luminosidade ideal (SILVA et al., 2020), o que também acontece para as espécies de plantas do gênero Amaranthus (CARVALHO; CHRISTOFFOLETI, 2007).

O malte é o produto da germinação controlada de qualquer cereal, tendo como objetivo a ativação e a produção de enzimas que atuarão na transformação do amido em açúcares, sendo a principal matéria-prima da cerveja (KUNZE, 2006). O processo de maltagem basicamente consiste de uma etapa inicial de umidificação dos grãos, denominado maceração, seguido de etapa controlada de germinação, onde se ativa o sistema enzimático. Após, 
quando o processo atinge um determinado ponto de germinação, o processo é cessado através da secagem.

O poder diastático é o parâmetro que mede a atividade da $\alpha$-amilases e $\beta$-amilases, ou seja, ele avalia a capacidade dessas enzimas em quebrar o amido; sendo um parâmetro de qualidade do malte (HOYLE et al., 2020). São relatados valores padrão para o poder diastático de no mínimo 220 WK; valores muito baixos podem gerar problemas na etapa de brasagem e os valores elevados podem influenciar o grau de fermentação (KUNZE, 2006).

Alguns autores pesquisaram sobre malte de amaranto (FERREIRA et al., 2007; NABUUMA et al., 2013; MOTTA et al., 2019), no entanto estudos ainda são escassos. Neste sentido, o presente trabalho teve como objetivo obter e avaliar o malte de diferentes variedades de amaranto (Amaranthus cruentus e Amaranthus caudatus), produzidas no cerrado mato-grossense.

\section{MATERIAL E MÉTODOS}

O trabalho foi desenvolvido na Universidade Federal de Mato Grosso, Cuiabá, Mato Grosso. A pesquisa foi realizada em duas etapas, sendo a primeira no Laboratório de Sementes para estudo da germinação e produção do malte. A segunda parte, onde determinou-se o poder diastático dos maltes produzidos, foi realizada no Laboratório de Físicoquímica de Alimentos.

Foram utilizadas sementes das espécies Amaranthus cruentus, com as variedades BRS Alegria e Verde, e Amaranthus caudatus, com uma variedade denominada Inca, produzidas na Fazenda Experimento da Universidade Federal do Mato Grosso, Santo Antônio do Leverger, Mato Grosso, coordenadas geográficas $15^{\circ} 50^{\prime} 48^{\prime \prime}$ S e $56^{\circ} 03^{\prime} 52^{\prime \prime} \mathrm{W}$, altitude de $140 \mathrm{~m}$.

A variedade BRS Alegria foi selecionada por sua adaptação ao clima do Cerrado brasileiro (SILVA et al., 2019). Suas sementes são de aproximadamente $1,0 \mathrm{~mm}$ e sãos ricas em aminoácidos essenciais. A variedade Verde é uma derivada da BRS Alegria, selecionada devido à coloração das nervuras dos cotilédones verdes, diferenciando-se do BRS Alegria de nervuras avermelhadas. A variedade denominada Inca, foi selecionada devido à suas características em relação a espécie, diferenciando-se das outras duas, e é uma variedade estudada na Faculdade de Agronomia e Zootecnia da UFMT desde 2009.

O teste de germinação foi realizado conforme a recomendação do Manual de Regras para a Análise de Sementes (BRASIL, 2009). As sementes passaram por um processo de limpeza, em que foram mergulhadas em solução de hipoclorito de sódio $(\mathrm{NaClO})$, por um minuto, e lavadas com água destilada antes de iniciar o processo de germinação para a evitar a contaminação.

Foram utilizadas caixas de germinação (gerbox), com substrato mata-borrão, utilizando-se 50 sementes espaçadas uniformemente. Para manter o ambiente controlado foi usada uma estufa incubadora tipo BOD (Biochemical Oxygen Demand) com controle eletrônico de temperatura e luminosidade, mantendo-se a temperatura em $25{ }^{\circ} \mathrm{C}$ (CARVALHO; CHRISTOFFOLETI, 2007) e exposição das sementes a luz controlada com três fotoperíodos, de 0,12 e 24 horas.

Para o teste de germinação, considerou-se delineamento inteiramente casualizado (DIC), com esquema fatorial 3x3, sendo três variedades de amaranto (BRS Alegria, Verde e Inca) e três fotoperíodos (0, 12 e 24 horas), com cinco repetições; utilizou-se o programa estatístico Assistat versão 7.7 pt para a análise dos dados.

O teste de germinação precedeu a produção de malte pois serviam de base para a maltagem, uma vez que eles avaliam as condições mais adequadas de luz e a viabilidade das sementes (KUNZE, 2006).

$\mathrm{O}$ experimento de produção de malte foi realizado em duas condições de temperatura, em $20^{\circ} \mathrm{C}$ (BRASIL, 2009) e $25{ }^{\circ} \mathrm{C}$ (CARVALHO; CHRISTOFFOLETI, 2007), para as três variedades de amaranto. A produção do malte envolveu o preparo da matéria prima, a maceração, a germinação, a secagem e a crivagem e foi obtida conforme o fluxograma adaptado de Phiarais e Arendt (2008).

As sementes foram pesadas, na quantidade de $200 \mathrm{~g}$, inseridas em uma embalagem de tecido com malha fina, conhecido como saco de filó, e passaram pelo processo de limpeza como já descrito. Para a maceração, ou embebição, utilizou-se uma bomba de ar de oxigênio, ou aerador, com as sementes suspensas através do saco, para que houvesse melhor ação do aerador, e foi adicionada água destilada suficiente para cobrir todas as sementes. A maceração foi realizada de acordo com o método das sementes submersas (BASKIN; BASKIN, 2014), com duração de 10 horas.

Para a fase de germinação da maltagem, as sementes maceradas foram espalhadas sobre bandejas forradas por papel manteiga e cobertas por filme PVC, para manter o ambiente saturado e reduzir a perda de água pelas sementes. Foi realizado revolvimento e umedecimento da massa de semente a cada 24 horas. As sementes ficaram nesse processo por $64 \mathrm{~h}$ para temperatura de $20^{\circ} \mathrm{C}$ e $48 \mathrm{~h}$ para a temperatura de $25^{\circ} \mathrm{C}$, em que se obteve a mesma taxa de germinação, com fotoperíodo de 12 horas, levando em consideração o teste de germinação realizado previamente.

Para a secagem, o malte verde foi distribuído sobre uma peneira com malha de $0,85 \mathrm{~mm}$, em aço inoxidável, e levado à estufa de circulação forçada à temperatura de $50{ }^{\circ} \mathrm{C}$ durante $36 \mathrm{~h}$. A crivagem foi realizada utilizando-se a mesma peneira, com a esfrega manual para a retirada das radículas.

Calculou-se o rendimento de acordo com a massa final e a massa inicial (200 g) do malte, considerando as perdas por umidade e processos germinativos. A umidade foi realizada pelo método direto, em estufa de circulação forçada, à 105 ${ }^{\circ} \mathrm{C}$, por $24 \mathrm{~h}$ (BRASIL, 2009). A qualidade dos maltes produzidos foi realizada por meio da análise do poder diastático, conforme metodologia da Analytica EBC (2006).

Para a produção de malte, considerou-se delineamento inteiramente casualizado (DIC), com esquema fatorial $3 \times 2$, sendo três variedades de amaranto (BRS Alegria, Verde e Inca) e duas temperaturas de maltagem $\left(20\right.$ e $\left.25^{\circ} \mathrm{C}\right)$, com três repetições. Os resultados foram submetidos à ANOVA e ao teste de Tukey, ou regressão não linear, conforme o caso, sempre que o teste " $F$ " apresentou significância.

\section{RESULTADOS}

$\mathrm{Na}$ Tabela 1 são apresentados os resultados do teste de germinação das sementes com diferentes fotoperíodos. Para todas sementes avaliadas, foi verificada germinação próxima da máxima, acima de $80 \%$ para o fotoperíodo de 12 horas. As menores taxas de germinação foram as obtidas nos tratamentos com presença de luz por 24 horas, com nível crítico para a variedade BRS Alegria, de 16\%.

Observou-se que $A$. cruentus var. Verde apresentou porcentagem de germinação elevada indiferentemente do tempo de exposição à luz, ou seja, germinam tanto na 
presença como em ausência de luminosidade, com valores acima de $90 \%$ (Tabela 1 ).

Tabela 1. Teste de germinação (\%) das sementes de Amaranthu. cruentus, var. BRS Alegria e Verde, e A. caudatus, var. Inca, com diferentes fotoperíodos.

Table 1. Seed germination test of Amaranthus cruentus, var. BRS Alegria and Verde, and $A$. caudatus, var. Inca, with different photoperiods.

\begin{tabular}{ccccc}
\hline \multirow{2}{*}{ Variedades } & \multicolumn{3}{c}{ Fotoperíodo } & \multirow{2}{*}{ Média } \\
\cline { 2 - 4 } & $0 \mathrm{~h}$ & $12 \mathrm{~h}$ & $24 \mathrm{~h}$ & \\
\hline BRS Alegria & $62,5 \mathrm{bB}$ & $80,0 \mathrm{aB}$ & $16,0 \mathrm{cC}$ & $52,8 \mathrm{C}$ \\
Verde & $97,5 \mathrm{aA}$ & $97,5 \mathrm{aA}$ & $90,0 \mathrm{aA}$ & $95,0 \mathrm{~A}$ \\
Inca & $91,0 \mathrm{aA}$ & $91,5 \mathrm{aA}$ & $44,5 \mathrm{bB}$ & $75,7 \mathrm{~B}$ \\
\hline Média & $88,1 \mathrm{a}$ & $85,7 \mathrm{a}$ & $50,7 \mathrm{~b}$ & \\
\hline
\end{tabular}

Médias seguidas de mesma letra minúscula, na linha, ou maiúscula, na coluna, não diferem estatisticamente (Tukey, $\mathrm{p}>0,05)$.

Means followed by the same lowercase letter, in the line, or uppercase, in the column, do not differ statistically (Tukey, $\mathrm{p}>0.05$ ).

Tanto a BRS Alegria, quanto a Inca, tiveram sua germinação reduzida pela presença de luz por 24 horas, de 16 e 44,5\%, respectivamente; ainda, a BRS Alegria apresentou uma queda na germinação quando em ausência de luminosidade, demonstrando a necessidade da alternância do fotoperíodo, enquanto que a Inca manteve sua germinação próxima a máxima (Tabela 1).

O resultado do rendimento obtido no processo de maltagem das diferentes variedades de amaranto estudadas se encontra na Tabela 2. Foram calculados os valores de umidade na proporção de $12,31 \%, 7,94 \%$ e $7,71 \%$ para BRS Alegria, $11,25 \%, 8,17 \%$ e 8,37\% para Verde e $10,67 \%, 7,60 \%$ e $7,75 \%$ para a Inca, para grãos, malte seco à $20{ }^{\circ} \mathrm{C}$ e malte seco à $25^{\circ} \mathrm{C}$, respectivamente.

Tabela 2. Rendimento (\%) do malte de Amaranthus cruentus, var. BRS Alegria e Verde, e $A$. caudatus, var. Inca, em duas diferentes temperaturas de maltagem.

Table 2. Yield (\%) of malt from Amaranthus cruentus, var. BRS Alegria e Verde, and $A$. caudatus, var. Inca, at two different malting temperatures.

\begin{tabular}{cccc}
\hline \multirow{2}{*}{ Variedades } & \multicolumn{2}{l}{ Temperatura de maltagem } & \multirow{2}{*}{ Média } \\
\cline { 2 - 3 } & $20{ }^{\circ} \mathrm{C}$ & $25^{\circ} \mathrm{C}$ & \\
\hline BRS Alegria & $83,5 \mathrm{aA}$ & $84,2 \mathrm{aA}$ & $83,8 \mathrm{~A}$ \\
Verde & $80,7 \mathrm{bB}$ & $85,6 \mathrm{aA}$ & $83,2 \mathrm{~A}$ \\
Inca & $82,7 \mathrm{aA}$ & $79,7 \mathrm{bB}$ & $81,2 \mathrm{~B}$ \\
\hline Média & 82,3 & 83,2 & \\
\hline
\end{tabular}

Médias seguidas de mesma letra minúscula, na linha, ou maiúscula, na coluna, não diferem estatisticamente (Tukey, $\mathrm{p}>0,05)$.

Means followed by the same lowercase letter, in the line, or uppercase, in the column, do not differ statistically (Tukey, $\mathrm{p}>0.05$ ).

Para a BRS Alegria, em ambas as temperaturas, o rendimento foi estatisticamente igual; para a Verde, o rendimento foi favorecido à temperatura de $25^{\circ} \mathrm{C}$, e, para a Inca, à $20^{\circ} \mathrm{C}$ se obteve maior rendimento. De forma geral, os rendimentos dos maltes produzidos foram maiores que $80 \%$, representando menos que $20 \%$ de perdas.

Em relação ao poder diastático do malte produzido, valores maiores foram encontrados à temperatura de $25^{\circ} \mathrm{C}$, independente da variedade; A Inca foi a que apresentou $\mathrm{O}$ maior poder diastático entre os materiais estudados, independente da temperatura. A BRS Alegria teve menor valor médio entre os tratamentos e a Verde ficou prejudicada à $20{ }^{\circ} \mathrm{C}$, tendo o menor valor de poder diastático entre os tratamentos (Tabela 3).
Tabela 3. Poder diastático (WK) do malte de Amarantbus cruentus, var. BRS Alegria e Verde, e A. caudatus, var. Inca, em duas diferentes temperaturas de maltagem.

Table 3. Diastatic power (WK) of malt from Amaranthus cruentus, var. BRS Alegria e Verde, and $A$. caudatus, var. Inca, at two different malting temperatures.

\begin{tabular}{cccc}
\hline \multirow{2}{*}{ Variedades } & \multicolumn{2}{c}{ Temperatura de maltagem } & \multirow{2}{*}{ Média } \\
\cline { 2 - 3 } & $20{ }^{\circ} \mathrm{C}$ & $25^{\circ} \mathrm{C}$ & \\
\hline BRS Alegria & $113,8 \mathrm{bB}$ & $140,2 \mathrm{aC}$ & $127,0 \mathrm{~B}$ \\
Verde & $100,3 \mathrm{bC}$ & $177,5 \mathrm{aB}$ & $138,9 \mathrm{~B}$ \\
Inca & $132,1 \mathrm{bA}$ & $187,4 \mathrm{aA}$ & $159,7 \mathrm{~A}$ \\
\hline Média & $115,4 \mathrm{~b}$ & $168,4 \mathrm{a}$ & \\
\hline
\end{tabular}

Médias seguidas de mesma letra minúscula, na linha, ou maiúscula, na coluna, não diferem estatisticamente (Tukey, $\mathrm{p}>0,05)$.

Means followed by the same lowercase letter, in the line, or uppercase, in the column, do not differ statistically (Tukey, $\mathrm{p}>0.05$ ).

\section{DISCUSSÃO}

A germinação acima de $80 \%$, encontrados no fotoperíodo de 12 horas, indica boa qualidade e viabilidade das sementes (BRASIL, 2009). Na ausência e presenta total de luz, a germinação não fica eficiente principalmente para a var. BRS Alegria. Em outras espécies do gênero Amaranthus (A.bybridus, $A$. retroflexus, $A$. viridis, $A$. deflexus e $A$. spinosus), maiores taxas e velocidades de germinação também foram obtidas com alternância de luminosidade (CARVALHO; CHRISTOFFOLETI, 2007).

No entanto, para a variedade Verde, esta alternância de luminosidade não fez diferença, mantendo germinação acima dos $90 \%$, portanto, esta pode ser denominada de fotoblástica neutra (BEWLEY et al., 2012). Da mesma forma, Silva et al. (2020) concluíram que a luz não foi um fator que promoveu variações na germinação das sementes de Macroptilium lathyroides, que pode germinar de forma satisfatória tanto na presença quanto na ausência de luz.

Para a variedade Inca, a ausência de luz permitiu uma alta germinação, o que indica que mesmo enterradas no solo, elas germinariam eficientemente. Lessa et al. (2013) relatam germinação mesmo na ausência de luminosidade em sementes de Emilia coccinea, afirmando que este pode ser um fator indicativo de fotoblastismo preferencial.

O processo de maltagem apresenta perdas de diferentes fontes de forma que a massa de malte produzida é inferior à massa de sementes que entraram no processo (PHIARAIS; ARENDT, 2008).

Os valores de poder diastático obtidos chegaram, em média, a $168,4 \mathrm{WK}$, à $25{ }^{\circ} \mathrm{C}$, inferior ao sugerido pela literatura de $220 \mathrm{WK}$ (KUNZE, 2006).

O malte de amaranto apresentou poder diastásico inferior e pode não ser capaz de realizar a conversão de todo amido presente nos grãos no processo de brasagem (KUNZE, 2006). Isto sugere que novos ensaios de maltagem devam ser conduzidos, na tentativa de elevar o poder diastásico do malte deste pseudocereal para valores próximos do mínimo recomendado para fins cervejeiros. No entanto, os resultados indicam que há possibilidade de uso de malte de amaranto para produção de bebidas maltadas.

Os resultados obtidos neste trabalho são semelhantes aos encontrados por Nabuuma et al. (2013), que conseguiram, em maltagem de amaranto (Amaranthus caudatus) até 72 horas, maiores quantidades de amido hidrolisado, da ordem de $46,35 \% \pm 4,31$, com malte obtido após 48 horas de germinação. 


\section{CONCLUSÕES}

As menores taxas de germinação foram obtidas nos tratamentos com presença de luz por 24 horas. As maiores germinações foram com fotoperíodo de 12 horas. A variedade Verde é fotoblástica neutra. A variedade Inca é fotoblástica positiva "preferencial". A BRS Alegria apresenta necessidade de alternância de luz.

Considerando as condições em que ocorreu a maltagem de Amaranthus cruentus, variedades BRS Alegria e Verde, e $A$. caudatus, variedades Inca: altos rendimentos foram encontrados, acima de $80 \%$; a temperatura de $25^{\circ} \mathrm{C}$ produziu o maior poder diastático, independente da variedade; $A$. caudatus var. Inca apresentou maior poder diastático, independente da temperatura; $A$. cruentus $\mathrm{cv}$. BRS alegria teve menor poder diastático, sendo menos indicada para a produção de malte de amaranto. A variedade Inca apresentou maior poder diastático dentre os materiais estudados, em $25^{\circ}$ C, de 187,4 WK.

\section{AGRADECIMENTOS}

Ao Conselho Nacional de Desenvolvimento Científico e Tecnológico $(\mathrm{CNPq})$ pelo financiamento desta pesquisa.

\section{REFERÊNCIAS}

ANALYTICA EBC. Diastatic Power of Malt. Method 4.12. Disponível em: <https://brewup.eu/ebcanalytica/malt/diastatic-power-of-malt-byspectrophotometry-manual-method/4.12.1>. Acesso em: 23 set 2021.

BASKIN, C. C.; BASKIN, J. M. Seeds: ecology, biogeography, and evolution of dormancy and germination. 2.ed. New York: Academic Press, 2014. 1600p.

BEWLEY, J. D.; BRADFORD, K.; HILHORST, H.; NONOGAKI, H. Seeds: physiology of development, germination and dormancy. 3 ed. New York: Springer, 2012. 392p.

BRASIL. Regras para Análise de Sementes/ Ministério da Agricultura, Pecuária e Abastecimento. Secretaria de Defesa Agropecuária. Brasília: Mapa/ACS, 2009. 398p.

CARVAlHO, S. J. P.; CHRISTOFFOLETI, P. J. Áreas básicas influência da luz e da temperatura na germinação de cinco espécies de plantas daninhas do gênero Amaranthus. Bragantia, v. 66, n. 4, p. 527-533, 2007. DOI: 87052007000400001

FERREIRA, T. A. P. C.; MATIAS, A. C. G.; ARÊAS, J. A. G. Características nutricionais e funcionais do Amaranto (Amaranthus spp.) Nutritional and functional characteristics of Amaranth (Amaranthus spp.). Nutrire: Revista da Sociedade Brasileira de Alimentação e Nutrição, v. 32, n. 2, p. 91-116, 2007.

HOYLE, A.; BRENNAN, M.; PITTS, N.; JACKSON, G. E.; HOAD, S. Relationship between specific weight of spring barley and malt quality. Journal of Cereal Science, v. 95, e103006, 2020. DOI: https://doi.org/10.1016/j.jcs.2020.103006
KUNZE, W. Tecnología para Cerveceros y Malteros. 1 ed. [s.l: s.n.], 2006. 1074 p.

LESSA, B. F. T., FERREIRA, V. M.; NETO, J. C. A.; SOUZA, R. C. Germinação de sementes de Emilia coccinea (Sims) G. DON em função da luminosidade, temperatura, armazenamento e profundidade de semeadura. Semina: Ciências Agrárias, v. 34, n. 6, p. 3193-3204, 2013. DOI: https://doi.org/10.5433/16790359.2013v34n6Supl1p3193

MOTTA, C.; CASTANHEIRA, I.; GONZALES, G. B.; DELGADO, I.; TORRES, D.; SANTOS, M.; MATOS, A. S. Impact of cooking methods and malting on amino acids content in amaranth, buckwheat and quinoa. Journal of Food Composition and Analysis, v. 76, p. 58-65, 2019.2 DOI: https://doi.org/10.1016/j.jfca.2018.10.001

NABUUMA, D.; NAKIMBUGWE, D.; BYARUHANG, Y. B.; SAALIA, F. K.; PHILLIPS, R. D.; CHEN, J. Development of the Process for a Drinkable Plant-Based Infant Food. Journal of Nutrition \& Food Sciences, Brussels, v. 3, n. 4, p. 1-7, 2013. DOI: https://doi.org/10.4172/2155-9600.1000210

PAGAMUNICI, L. M.; SOUZA, A. H. P.; GOHARA, A. K.; SOUZA, N. E.; GOMES, S. T. M.; MATSUSHITA, M. Development, characterization and chemometric analysis of a gluten-free food bar containing whole flour from a new cultivar of amaranth. Ciência e Agrotecnologia, Lavras, v. 38, n. 3, p. 270-277, 2014. DOI: https://doi.org/10.1590/S1413-70542014000300007

PHIARAIS, B. P. N.; ARENDT, E. K. Malting and brewing with gluten-free cereals. In: ARENDT, E. K.; DAL BELLO, F. (Ed.). Gluten-Free Cereal Products and Beverages. [s.l: s.n.], 2008. p. 347-372.

SILVA, J. G.; BIANCHINI, A.; CRIVELARI-COSTA, P. M.; LOBO, F. A.; ALMEIDA, J. P. M.; MORAES, M. F. Amaranth Response to Water Stress. Journal of Experimental Agriculture International, v. 40, n. 1, p. 1-9, 2019.2 DOI: https://doi.org/10.9734/JEAI/2019/v40i130356

SILVA, M. S. A.; YAMASHITA, P. O. M.; ROSSI, A. A. B.; CARVALHO, M. A. C.; CONCENÇO, G.; SÁ, M. E. Influence of light and temperature on seed germination of Macroptilium lathyroides. South American Journal of Basic Education, Technical and Technological, [S. 1.], v. 7, n. 1, p. 311-324, 2020.

ZHANG, Z.S.; KANG, Y.J.; CHE, L. Composition and thermal characteristics of seed oil obtained from Chinese amaranth. LWT-Food Science and Technology, v. 111, p. 39-45, 2019. DOI: https://doi.org/10.1016/j.lwt.2019.05.007 